\title{
An Alternative perspective to Riemann Hypothesis
}

\author{
Jamal Y. Mohammad Salah ${ }^{1}$ \\ ${ }^{1}$ Department of Basic Science, College of Health and Applied Sciences,A’Sharqiyah University, Ibra. \\ Email:12damous73@yahoo.com
}

\begin{abstract}
We review some main results of Riemann Zeta function; the Integral representation the analytic continuity and the first functional equation by the means of Gamma function and Hankel contour. We observe that an additional term is considered in both results. We justify the non-trivial location of Zeta non-trivial zeros subject to an approximation.
\end{abstract}

Keywords

Riemann Hypothesis, Analytic Continuity, Functional Equation, Hankel Contour.

Article Received: 10 August 2020, Revised: 25 October 2020, Accepted: 18 November 2020

\section{Introduction}

In 1859, Bernhard Riemann published an eight-page paper, in which he estimated "the number of prime numbers less than a given magnitude" using a certain meromorphic function on $\mathbb{C}$. But Riemann did not fully explain his proofs; it took decades for mathematicians to verify his results, and to this day we have not proved some of his estimates on the roots of $\zeta$. Even Riemann did not prove that all the zeros of $\zeta$ lie on the line $\operatorname{Re}(s)=1 / 2$. This conjecture is called the Riemann hypothesis and is considered by many the greatest unsolved problem in mathematics $[1,3,5,7]$

The difference between the analytic continuity of Gamma and Zeta

$\zeta(s)=\sum_{n=1}^{\infty} n^{-s}, \quad \operatorname{Re}(s)>1$
$\Gamma(s)=\int_{0}^{\infty} e^{-\tau} \tau^{s-1} d \tau, \quad \operatorname{Re}(s)>1$.

Clearly, $\quad \tau=0$ is the lower limit of the integral representation of $\Gamma(s)$. Now, considering the Hankel Contour approach we can certainly provide the analytic continuity for all $s$ except $s=0,-1,-2 \ldots$ see $[2,3,4$ and 10] Riemann considered the same approach $[1,2,3$ and 10]

Let $\tau=n t \rightarrow d \tau=n d t$ yields

$\Gamma(s)=n^{s} \int_{0}^{\infty} e^{-n t} t^{s-1} d t$

$\zeta(s) \Gamma(s)=\sum_{n=1}^{\infty} \int_{0}^{\infty} e^{-n t} t^{s-1} d t=\int_{0}^{\infty}\left(\sum_{n=1}^{\infty} e^{-n t}\right) t^{s-1} d t=\int_{0}^{\infty} \frac{t^{s-1}}{e^{t}-1} d t$,

Now, since we already assumed the limit of the geometric series

$\sum_{n=1}^{\infty} e^{-n t}=\frac{1}{e^{t}-1}, n \rightarrow \infty$

We may wish to reconsider that

$\tau=n t$ and $n \rightarrow \infty \Rightarrow t \neq 0$. Otherwise we are

letting $\tau=\infty \times 0$ which is undefined

In view of that; obviously $t=0$, is not a lower limit of the integral representation of $\zeta(s)$. Consequently; $\zeta(s) \Gamma(s)=\int_{0}^{\infty} \frac{t^{s-1}}{\left(e^{t}-1\right)} d t, t \neq 0, \quad n$ already $\left.\rightarrow\right)^{\infty}$

Through the representation above, the one cannot provide any analytic continuity by the means of Hankel contour since there will be always a gap around $t=0$.

If we assume that

$\zeta(s) \Gamma(s)=\int_{0}^{\infty} \frac{t^{s-1}}{\left(e^{t}-1\right)} d t$.

\section{We are assuming an additional term around $t=0$}

For that, we may expect some accurate conclusions but not sharp. A straight forward approach toward locating the nontrivial zeros can be considered from the following observation:

For a non zero real valued number $t$ if

$t^{s-1}+t^{-s}=0 . \quad t \neq 0, \quad$ then

$s=\frac{1}{2}+i \frac{(2 m+1) \pi}{2 \ln t}, \quad t \neq 0, \ln t \neq 0$ and $m \in \mathbb{Z}$

That is if we assume the analytic continuity from (1.1) and if we let $\zeta(s)=\zeta(1-s)=0$.

Then

$\zeta(s) \Gamma(s)+\zeta(1-s) \Gamma(1-s)=\int_{0}^{\infty} \frac{t^{s-1}+t^{-s}}{\left(e^{t}-1\right)} d t=0, \quad t \neq 0$

We can let the right hand side equal zero elements wise $\operatorname{Re}(s)>1 \cdot t^{s-1}+t^{-s}=0$.

$s=\frac{1}{2}+i \frac{(2 m+1) \pi}{2 \ln t}, \quad t \neq 0$, lnt $\neq 0$ and $m \in \mathbb{Z}$

This implies that

The non-trivial zeros lie on the critical line $\operatorname{Re}(s)=\frac{1}{2}$ Next;

We consider the Hankel Contour that is we assume the non sharp version (1.2). We briefly state and prove the analytic continuity and the first functional equation

Riemann Zeta FunctionIntegral Formula and First Functional Equation [1 - 12] 
Lemma 1the Riemann Zeta function is meromorphic everywhere, except at a simple pole $s=1$

Proof

$\Gamma(s)=\int_{0}^{\infty} e^{-\tau} \tau^{s-1} d \tau=n^{s} \int_{0}^{\infty} e^{-n t} t^{s-1} d t, \quad \tau=n t$.

Multiplying by $\zeta(s)_{x}$ implies

$\zeta(s) \Gamma(s)=\int_{0}^{\infty} \frac{t^{s-1}}{e^{t}-1} d t . \quad \operatorname{Re}(s)>0$.

To extend this formula to $\mathbb{C}\{1\}$, we integrate $(-t)^{s} /\left(e^{t}-1\right)$ over a Hankel contour: a path from $+\infty$ inbound along the real line to $\epsilon>0$, counterclockwise around a circle of radius $E$ at 0 , back to $E$ on the real line, and outbound back to $+\infty$ along the real line, around the circle, $t$ can be parameterized by $t=\epsilon e^{i \theta}, 0 \leq \theta \leq 2 \pi$ and $\epsilon$ is a small arbitrary positive constant that we will let tend to 0 :

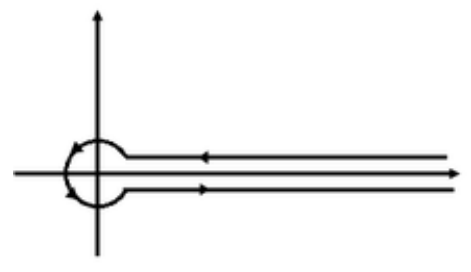

Figure 1. Hankel Contour

$\oint_{\sigma} \frac{(-t)^{s-1}}{e^{t}-1} d t=\int_{\rho_{1}} \frac{(-t)^{s-1}}{e^{t}-1} d t+\int_{\rho} \frac{(-t)^{s-1}}{e^{t}-1} d t+\int_{\rho_{2}} \frac{(-t)^{s-1}}{e^{t}-1} d t$ $=\int_{R}^{\epsilon} \frac{\left(t e^{-\pi i}\right)^{s-1}}{e^{t}-1} d t+i \epsilon \int_{0}^{2 \pi} \frac{\left(\epsilon e^{-\pi i} e^{i \theta}\right)^{s-1}}{e^{\epsilon e^{i \theta}}-1} e^{i \theta} d \theta+e^{2 \pi i} \int_{\epsilon}^{R} \frac{\left(t e^{-\pi i} e^{2} \text { depend on the path. The only significant note is the integral }\right.}{e^{t e^{2 \pi i}}-1} d$ and small circle vanishes subject to $\epsilon=0$.

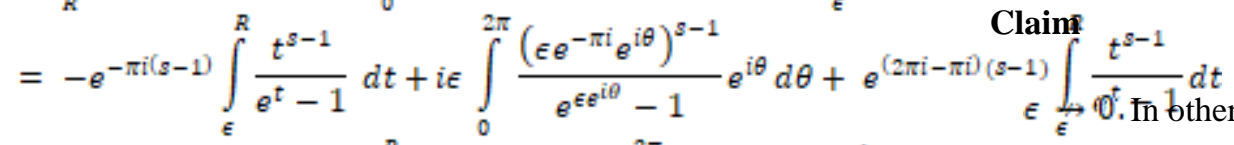

$=\left(e^{\pi i(s-1)}-e^{-\pi i(s-1)}\right) \int_{\epsilon}^{R} \frac{t^{s-1}}{e^{t}-1} d t+i \epsilon \int_{0}^{2 \pi} \frac{\left(e e^{-\pi i} e^{i \theta}\right)^{s-1}}{e^{\epsilon e^{i \theta}}-1} e^{i \theta} d \theta$

$=2 i \sin (\pi s) \int_{\epsilon}^{R} \frac{t^{s-1}}{e^{t}-1} d t+i \epsilon \int_{0}^{2 \pi} \frac{\left(\epsilon e^{-\pi i} e^{i \theta}\right)^{s-1}}{e^{\epsilon e^{i \theta}}-1} e^{i \theta} d \theta$

$\lim _{\substack{E \rightarrow 0 \\ R \rightarrow \infty}}\left[2 i \sin (\pi s) \int_{\epsilon}^{R} \frac{t^{g-1}}{e^{t}-1} d t+i \epsilon \int_{0}^{2 \pi} \frac{\left(\epsilon e^{-\pi i} e^{i \theta}\right)^{g-1}}{e^{\epsilon e^{2 \theta}}-1} e^{i \theta} d \theta\right]$.

$=2 i \sin (\pi s) \int_{0}^{\infty} \frac{t^{s-1}}{e^{t}-1} d t$

Finally;

$\oint_{c} \frac{(-t)^{s-1}}{e^{t}-1} d t=2 i \sin (\pi s) \Gamma(s) \zeta(s)$

Lemma 2
$\zeta(s)=2^{s} \pi^{s-1} \sin \left(\frac{\pi s}{2}\right) \Gamma(1-s) \zeta(1-s)$

\section{Proof}

Here we consider a modified Hankel contour (figure 2): consisting of two circles centered at the origin and a radius segment along the positive reals. The outer circle has radius $(2 n+1) \pi$ and the inner circle has radius $\varepsilon<\pi$. The outer circle is traversed clockwiseand the inner one counterclockwise. The radial segment is traversed in both directions. Then by employing the residue theorem

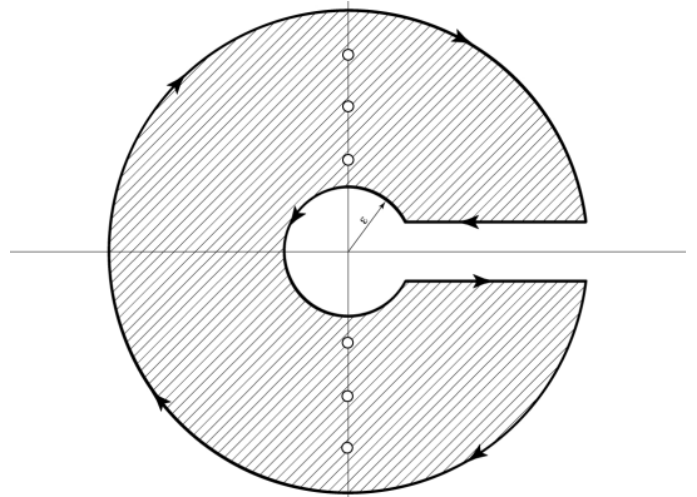

Figure 2

$\oint_{F} \frac{(-t)^{s-1}}{e^{t}-1} d t=2^{s} \pi^{s-1} \sin \left(\frac{\pi s}{2}\right) \sum_{n=1}^{\infty} n^{s-1}$

Plugging in equation (1.3) we then prove the functional equation

$\zeta(s)=2^{s} \pi^{s-1} \sin \left(\frac{\pi s}{2}\right) \Gamma(1-s) \zeta(1-s)$

Remark $^{[9,10]}$

From Lemma 1, the integral along the real axis in both directions does not depend on $\epsilon$. Similarly; the integral along the modified Hankel contour in Lemma 2 does not ther words $\epsilon$ will remain non-zero no matter how small it is.

\section{Proof}

Certainly; there exist a connection between $t$ and $\epsilon$, since $t$ can be parametrized around the small circle by $t=e e^{i \theta}{ }_{x}$ this implies $|t|=\epsilon \neq 0 \quad$ (since $\tau=n t, n \rightarrow \infty$ ).

Re Agcording to our claim, we will keep track on $E$ along the steps of the proofs of Lemma 1 and Lemma 2. We will let the integral around the circle.

$t=\epsilon e^{i \theta} \Rightarrow i \epsilon \int_{0}^{2 \pi} \frac{\left(\epsilon e^{-\pi i} e^{i \theta}\right)^{s-1}}{e^{\epsilon \theta^{\theta \theta}}-1} e^{i \theta} d \theta$
$i \epsilon \int_{0}^{2 \pi} \frac{\left(\epsilon e^{-\pi i} e^{i \theta}\right)^{s-1}}{e^{\epsilon e^{i \theta}}-1} e^{i \theta} d \theta \rightarrow f(s, \epsilon) \epsilon^{s-1} \rightarrow \epsilon^{s-1}$

For shorthand and since the function $f(s, \epsilon)$ will not directly contribute in our approach, we can simply omit it. Now, we consider the slight modification on Lemma 1 and Lemma 2, due to the additional term $\varepsilon^{\delta-1}$, the result in (1.3) will reduce to

$\oint_{\sigma} \frac{(-t)^{s-1}}{e^{t}-1} d t=e^{s-1}+2 i \sin (\pi s) \Gamma(s) \zeta(s)$. 
Similarly, the functional equation in Lemma 2 will be also modified and viewed asnon functional equations. Since we assumed the additional terms of $\varepsilon$ we have to add restrictions for accuracy.

$$
\begin{aligned}
& \zeta(s)=\epsilon^{s-1}+2^{s} \pi^{s-1} \sin \left(\frac{\pi s}{2}\right) \Gamma(1-s) \zeta(1-s) \\
& \zeta(1-s)=\epsilon^{-s}+2^{1-s} \pi^{-s} \sin \left(\frac{\pi(1-s)}{2}\right) \Gamma(s) \zeta(s)
\end{aligned}
$$

Theorem assuming the analytic continuity of $\zeta(s)$ and

\section{Proof}

$$
\text { If } \zeta(s)=\zeta(1-s)=0, \text { then } \operatorname{Re}(s)=\frac{1}{2}
$$

For simplicity we write

$$
\begin{aligned}
& A(s)=2^{s} \pi^{s-1} \sin \left(\frac{\pi s}{2}\right) \Gamma(1-s) \zeta(1-s) \\
& A(1-s)=2^{1-s} \pi^{-s} \sin \left(\frac{\pi(1-s)}{2}\right) \Gamma(s) \zeta(s)
\end{aligned}
$$

Let $s=x+i y$ satisfying $\zeta(s)=\zeta(1-s)=0$, then

$\zeta(s)-A(s) \zeta(1-s)=e^{s-1}$

$\zeta(1-s)-A(1-s) \zeta(s)=e^{-s}$.

Adding the last two expressions yield

$\zeta(s)-A(s) \zeta(1-s)+\zeta(1-s)-A(1-s) \zeta(s)=\epsilon^{s-1}+\epsilon^{-\sigma}$

Now, if the left hand side of the expression above equals zero implies the right hand side also equals zero, solving for $s \in \mathbb{C}\{1\}$ :

$e^{s-1}+e^{-s}=0$

$y=\frac{i \text { lne }(2 x-1)+(2 m+1) \pi}{2 \ln e}, \quad \epsilon \neq 0, \quad$ lne $\neq 0$ and $m \in \mathbb{Z}$

Since $y$ is real valued then the term multiplied by $i$ will vanish that is $x=\frac{1}{2}$, or if we write

$s=x+i\left[\frac{i \text { lne }(2 x-1)+(2 m+1) \pi}{2 l n e}\right]$

That is

$s=x+i\left[\frac{(2 m+1) \pi}{2 \ln e}\right]$

The real part of $s$ will reduce to $x=\frac{1}{2}$

\section{Consequences}

For $E$ sufficiently small; we can consider the following

$$
\begin{aligned}
& \zeta(s)=e^{s-1}+2^{s} \pi^{s-1} \sin \left(\frac{\pi s}{2}\right) \Gamma(1-s) \zeta(1-s), \quad F(s)>1_{x}
\end{aligned}
$$$$
\zeta(1-s)=e^{-s}+2^{1-s} \pi^{-s} \sin \left(\frac{\pi(1-s)}{2}\right) \Gamma(s) \zeta(s) .
$$$$
\left.{ }_{7}\right)^{R e}(s)<0
$$

This manuscript has not been submitted to, nor is under review at, another journal or other publishing venue.

We justified the location of the non-trivial zeta zeros subject to assuming the analytical continuity and subject to considering $\quad \zeta(s)=\zeta(1-s)=0 \quad$ simultaneousily. However this does not change the fact that the analytical continuity is not sharp nor all of its consequences: the functional equation, the trivial zeros and the non trivial zeros. The only sharp result is the location of non-trivial zeros on the critical line subject to assuming an approximated analytic continuity.

But since the one can manipulate the error, Riemann Zeta function remains an accurate approach to investigate the main objective of Riemann: the distribution of prime numbers.

\section{Conflicts of Interest}

\section{Funding Statement}

The author received no financial support for the research, and publication of this article

\section{Acknowledgments}

I express my gratitude to the Almighty for giving me strength and courage during the process of research writing and completion of this piece of work. I extend my gratitude to all researchers who provided a holistic approach to understand Riemann Hypothesis. The acknowledgments cannot be complete without thanking Stephen Wolfram and

$\zeta(s)=\epsilon^{s}+2^{s} \pi^{s-1} \sin \left(\frac{\pi s}{2}\right) \Gamma(1-s) \zeta(1-s), \quad 0<\frac{(1 e}{8}(s)<1$ www.wolframalpha.com

$\zeta(1-s)=\epsilon^{1-s}+2^{1-s} \pi^{-s} \sin \left(\frac{\pi(1-s)}{2}\right) \Gamma(s) \zeta(s), \quad(d<\operatorname{Re}(s)<1$.

B. Riemann, Uber die Anzahl der Primzahlen unter eine gegebene Grosse 1859

$\zeta(s)+\zeta(1-s)=0$, at $s=\frac{1}{2}+i y$.

$y=\frac{(2 m+1) \pi}{2 \ln t}$, for some non zero real number $t, m \in \mathbb{Z}$

[2] Bombieri, Enrico (2000), The Riemann Hypothesis - official problem description (PDF), Clay Mathematics Institute, retrieved 2008-10-25

$\zeta(-2 n) \sim 0, \quad n \in \mathbb{Z}$. 
[3] Borwein, Peter; Choi, Stephen; Rooney, Brendan; Weirathmueller, Andrea, eds. (2008), The Riemann Hypothesis: A Resource for the Afficionado and Virtuoso Alike, CMS Books in Mathematics, New York: $\quad$ Springer, doi:10.1007/978-0-38772126-2, ISBN 978-0-387-72125-5

[4] Harold M. Edwards. Riemann's Zeta Function. 1974. Dover Publications.

[5] H. Iwaniec Lectures on the Riemann zeta function, vol. 62 of University Lecture Series, American Mathematical Society, Providence, RI, 2014.

[6] Jekel, David. IThe Riemann Zeta Function." The University of Washington. n.p., 6 June 2013. Web.

[7] Https: ==www: math: Washington: Edu= morrow $=33613=$ papers $=$ david:pdf

[8] J. E. Littlewood The Riemann Hypothesis TheScientist Speculates, New York: Basic books, 1962

[9] E. C. Titchmarsh, The theory of the Riemann zeta-function, The Clarendon Press, Oxford University Press, New York, second ed., 1986. Edited and with a preface by D. R. Heath-Brown

[10] Jamal Y. Mohammad Salah, The consequence of the analytic continuity of Zeta function subject to an additional term and a justification of the location of the non-trivial zeros, International Journal of Science and Research (IJSR). Volume 9, Issue 3, Pages 1566 - 1569. 2020.

[11] Jamal Y. Mohammad Salah, Two Conditional proofs of Riemann Hypothesis, International Journal of Sciences: Basic and Applied Research (IJSBAR). Volume 49, Issue 1, Pages 7483. 2020.

[12] S. J. Patterson, An introduction to the theory of the Riemann zeta function, vol. 14 of Cambridge Studies in Advanced Mathematics, Cambridge University Press, Cambridge, 1988.

[13] Shigeki Akiyama, Shigeki Egami, and Yoshio Tanigawa. Analytic continuation of multiple zeta-functions and their values at non-positive integers. Acta ArithmeticaWarszawa, 98(2):107-116, 2001. 